\title{
The Pressure Drop Model of Liquid Flow with Wall Mass Transfer in Horizontal Wellbore with Perforated Completion
}

\author{
Ping Yue, Zhimin Du, Xiaofan Chen, and Chao Tang \\ The State Key Laboratory of Oil \& Gas Reservoir Geology and Exploitation Engineering, Southwest Petroleum University, \\ Chengdu, Sichuan 610500, China
}

Correspondence should be addressed to Ping Yue; yuepingaa@126.com

Received 16 December 2013; Revised 27 March 2014; Accepted 3 April 2014; Published 16 April 2014

Academic Editor: Marcelo M. Cavalcanti

Copyright (C) 2014 Ping Yue et al. This is an open access article distributed under the Creative Commons Attribution License, which permits unrestricted use, distribution, and reproduction in any medium, provided the original work is properly cited.

\begin{abstract}
The fluids in horizontal wells can exhibit complicated flow behaviors with wall mass transfer, partly due to the interaction between the main flow and the radial influx along the wellbore and the completion parameters used. This paper presents a novel regression model established based on the experiment data retrieved from the available literatures to determine the apparent friction factor for a single phase wellbore flow. The proposed model has the potential to be readily applicable to different perforation parameters, such as shot phasing and shot density. Compared with other models in the same practical example which is offered by Ouyang et al., the model of this paper to calculate the wellbore pressure is applicable and reasonable. This new model can be easily incorporated into reservoir simulators or analytical reservoir and horizontal wellbore inflow coupling models.
\end{abstract}

\section{Introduction}

Dikken [1] in 1989 proposed the first model to evaluate the production performance of a horizontal well with the consideration of the wellbore pressure drop. Since then, $[2$, 3] active research work has been carried out on horizontal wellbore pressure drop using experimental [4-8], analytical [9-12], and simulation methodologies. Asheim et al. [7] made a study on friction factor correlation for horizontal wellbore, which included accelerational pressure drop caused by the continuous fluid inflow along the wellbore. They stated that both wall friction and radial influx acceleration contributed to the total pressure drop along a perforated pipe and pointed out the wall friction factor could be calculated in the same way as for a regular, unperforated pipe. Su and Gudmundsson [13] conducted a research based on the experiments made on a perforated pipe geometrically similar to a wellbore casing (12SPF, $60^{\circ}$ phasing). The research outcome revealed that, under the experiment environment, the total pressure drop was induced by the wall friction, mixed effects such as perforation roughness and acceleration and the percentage is around $80 \%, 15 \%$, and $5 \%$, respectively. Another model presented by Ouyang et al. [14] in 2001 is a single phase wellbore flow model. The research considered more pressure drop factors. The model incorporated not only the effects from the friction, acceleration, and gravitation but also the pressure drop caused by radial inflow. Yuan et al. $[5,15]$ conducted an experimental investigation and theoretical analysis on flow behavior in a horizontal pipe with fluid injection from the pipe wall through a single injection point. Later on, they [16] used the same experimental facility to investigate the effects of completion geometries and the density and phasing of injection openings in horizontal wells. In their experiments, there were three test sections with perforation densities of 5,10 , and 20 shots per feet and phasing of $360^{\circ}$, $180^{\circ}$, and $90^{\circ}$. Jiang et al. [17] also used the same experimental facility to investigate other sections of perforation densities of 5,10 , and 20 shots per feet with phasing of $360^{\circ}, 180^{\circ}$, and $90^{\circ}$. Based on the data acquired from their experiments and the data obtained by Yuan, Jiang et al. found the three parameters of general apparent friction factor correlation through regression, which was developed from the principles of conservation of mass and conservation of momentum. However, the regression was limited to single completion section. Their attempt to develop correlations for parameters using perforation density and phasing as continuous variables was restricted due to the limited data to perform meaningful regression analysis $[17,18]$. 
Literature study revealed that although single phase pipe flow with wall mass transfer has been a hot research topic for both experimental study and theoretical analysis, there is not a formula that is able to depict the friction factor or apparent friction factor for the continuous transformation of perforation shot density and phasing. Based on the experimental data of Yuan and partial data of Jiang, this research developed a new correlation for predicting the apparent friction factor of horizontal wellbore with different completion shot densities, phasing, main flow Reynolds numbers, and radial influx rates.

\section{Model Description}

The approach developed by Yuan was adopted in this study to analyze the acquired data and to predict horizontal well friction factors for different shots completion geometries. The detailed derivation process can be found in Yuan et al. $[15,16]$.

An apparent friction factor, defined as the ratio of the net imposed external forces to the inertial forces, can be expressed as

$$
f_{t}=-\frac{\left(\left(p_{2}-p_{1}\right) / \Delta x\right)}{\left(\rho \bar{u}^{2} / 2 d\right)} .
$$

According to experiment data $\Delta p\left(p_{2}-p_{1}\right)$, the pipe parameters, main flow rate, influx flow rate, and liquid density, the expression for the apparent friction factor in Yuan's theoretical model can then be written as

$$
f_{t}=f_{w}+2 d\left(\frac{\beta_{2}-\beta_{1}}{\Delta x}\right)+2 d \varphi \frac{q}{\bar{Q}} C_{n} .
$$

The second term on the right-hand side of $(2), 2 d\left(\left(\beta_{2}-\right.\right.$ $\left.\beta_{1}\right) / \Delta x$ ), is caused by the change in velocity profile in the main flow direction. However, Yuan pointed out that this term, at small injection rate, was negligible since the small rate injection would not affect the velocity field significantly except in the near-wall region. Under what circumstances, can the value of $2 d\left(\left(\beta_{2}-\beta_{1}\right) / \Delta x\right)$ be ignored? Up until now, no successful attempt has been made to evaluate this term. Using Blasius formula $f_{w}=a N_{\mathrm{Re}}^{b}$, we can rewrite $f_{t}$ :

$$
f_{t}=a N_{\mathrm{Re}}^{b}+2 d \varphi C_{n} \frac{q}{Q}
$$

where $C_{n}, a$, and $b$ can be determined experimentally for different completion cases.

Jiang et al. [17] attempted to develop correlations for $a, b$, and $C_{n}$ using perforation shot density and phasing as parameters of interests by employing an additional regression analysis. However, they only just listed $a, b$, and $C_{n}$ in 9 cases for different combinations of perforation shot densities and phasing, respectively, through the method of regression. And there exist disagreements between Jiang's result and Yuan's outcome, such as the case where $\theta=90^{\circ}, \varphi=20$ shot $/ \mathrm{ft}$. This research made a study that combined Yuan's experimental data with part of the data obtained by Jiang's research. The data analysis and regress revealed that, regardless of the influx

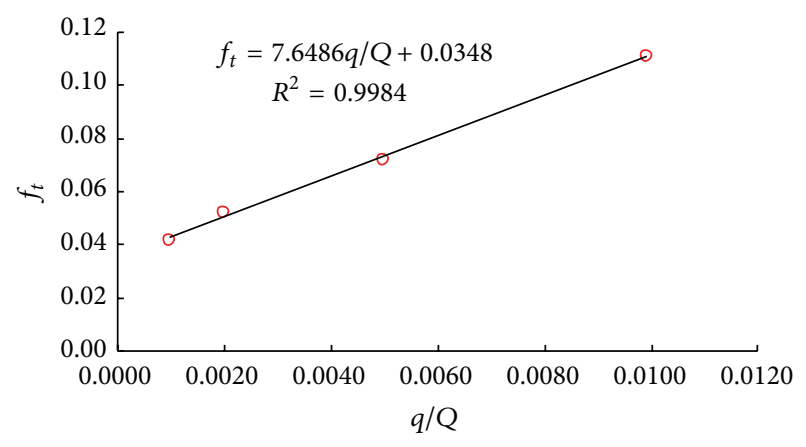

FIGURE 1: The relation between $f_{t}$ and $q / Q$ based on the experiment data (20 shots/ft, $\left.90^{\circ}, N_{\mathrm{Re}}=5000\right)$.

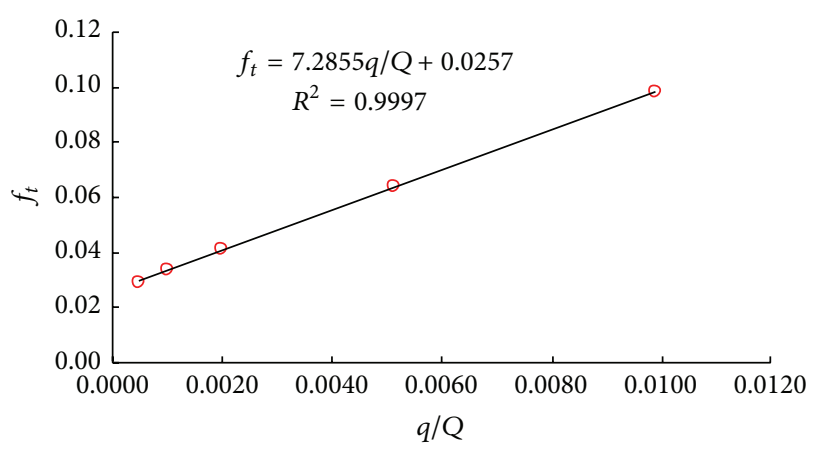

FIGURE 2: The relation between $f_{t}$ and $q / Q$ based on the experiment data (20 shots $\left./ \mathrm{ft}, 90^{\circ}, N_{\mathrm{Re}}=10000\right)$.

to main flow rate ratios, the $C_{n}$ for each case that all the data collapse into one curve, can be expressed by the variables of shot density and phasing. $C_{n}$ can be obtained through the following process.

Simplifying (3) gives

$$
f_{t}=B+A \frac{q}{Q} .
$$

From the experiments that were given the same shot density and phasing (such as $20 \mathrm{~s} / \mathrm{ft}, 90^{\circ}$ ) and main flow rate, by changing each shot radial influx to main flow rate ratio, we obtained the single factor sensitive analysis result and found that $f_{t}$ and $q / Q$ had a good linear relation. Therefore, parameter $A$ for each case was obtainable and is shown in Table 2. Finally, we used Blasius formula to regress the parameters $a$ and $b$ to define $B$ in $B=a N_{\mathrm{Re}}^{b}$ and the values are presented in Table 2.

\section{Data Analysis and Regression}

Chose Yuan's 20 shot $/ \mathrm{ft}, 90^{\circ}$ phasing experiment data analysis and regression, when given different main flow rate, which means different $N_{\mathrm{Re}}$, the relationship between $f_{t}$ and $q / Q$ in different main flow Reynolds Numbers are shown in Figures $1,2,3$, and 4 .

Figures 1-4 clearly show that $f_{t}$ and $q / Q$ have a good linear relation. Figure 5 indicates that the line slope (parameter $A$ ) decreases, while the main flow rate (parameter $N_{\mathrm{Re}}$ ) 
TABLE 1: The experiment data (20 shots $\left./ \mathrm{ft}-90^{\circ}\right)$ and regression parameters $A$ and $B$.

\begin{tabular}{cccccccccccccc}
\hline \multicolumn{1}{c}{ Radial influx rate to main flow rate ratio } & \multicolumn{4}{c}{ Radial influx rate to main flow rate ratio } \\
$N_{\mathrm{Re}}$ & $1 / 100$ & $1 / 200$ & $1 / 500$ & $1 / 1000$ & $1 / 2000$ & $A$ & $1 / 100$ & $1 / 200$ & $1 / 500$ & $1 / 1000$ & $1 / 2000$ & Average $B$ \\
& $f_{t}$ & $f_{t}$ & $f_{t}$ & $f_{t}$ & $f_{t}$ & & $B_{1}$ & $B_{2}$ & $B_{3}$ & $B_{4}$ & $B_{5}$ \\
\hline 5000 & 0.1108 & 0.0718 & 0.037 & 0.0416 & $/$ & 7.6486 & 0.04 & 0.0362 & 0.0374 & 0.0344 & $/$ \\
10000 & 0.0981 & 0.0612 & 0.0262 & 0.0332 & 0.029 & 7.2855 & 0.0273 & 0.0257 & 0.0266 & 0.0261 & 0.0254 & 0.0370 \\
20000 & $/$ & 0.0551 & 0.0199 & 0.0274 & 0.0233 & 7.0643 & $/$ & 0.0196 & 0.0199 & 0.0203 & 0.0197 & 0.0199 \\
40000 & $/$ & $/$ & 0.0149 & 0.022 & 0.0194 & 6.0052 & $/$ & $/$ & 0.014 & 0.0148 & 0.0158 & 0.0149 \\
\hline
\end{tabular}

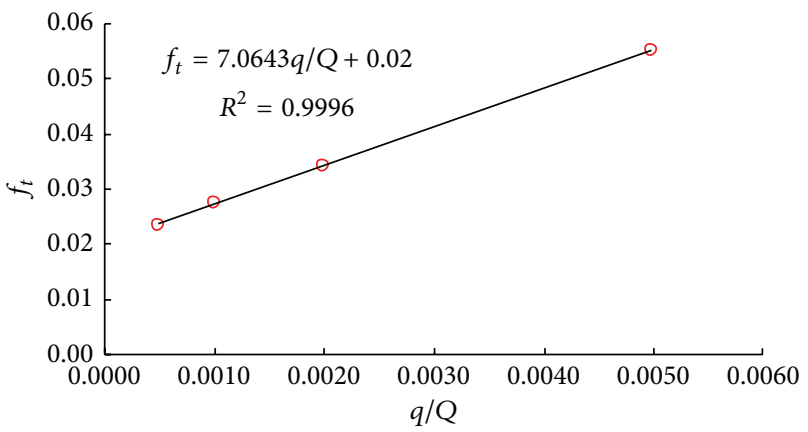

Figure 3: The relation between $f_{t}$ and $q / Q$ based on the experiment data (20 shots $\left./ \mathrm{ft}, 90^{\circ}, N_{\mathrm{Re}}=20000\right)$.

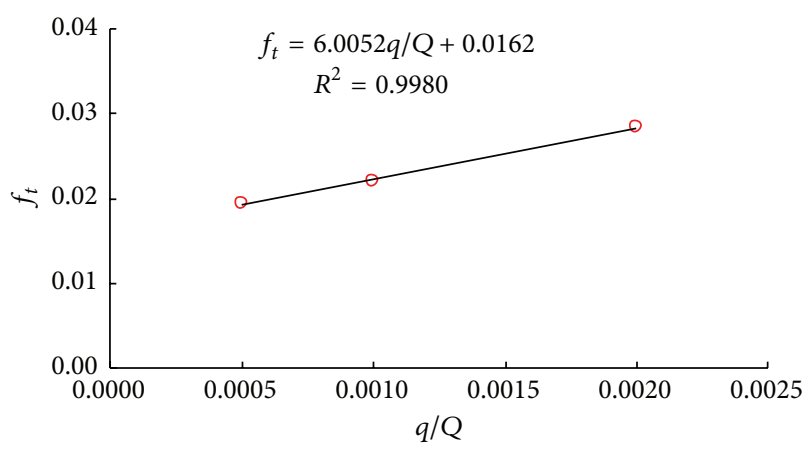

Figure 4: The relation between $f_{t}$ and $q / Q$ based on the experiment data (20 shots/ft, $\left.90^{\circ}, N_{\mathrm{Re}}=40000\right)$.

increases. In both Yuan and Jiang's regression processes, $A$ was made constant. Figure 5 shows that $A$ only changes a little if main flow rate does not change too much. When main flow rate is small, $N_{\mathrm{Re}}=5000$ and $A=7.65$ as shown in Figure 1. When main flow rate takes large value, $N_{\mathrm{Re}}=40000$ and $A=6.00$ as presented in Figure 4. The value of $A$ changes and the average value of $A$ is 7.14. This indicates that it is possible that, when main flow rate is too small or too big, the experiment facility has a little inaccuracy. Continue to follow Yuan and Jiang's principles and let the average value 7.14 be constant value of $A$ for the case $\left(20\right.$ shots $\left./ \mathrm{ft}, 90^{\circ}\right)$, which is correct and reasonably explained in the next paragraph.

When parameter $A=7.14$ is confirmed, then we can get the parameter $B$. The process to regress the relation between $B$ and $N_{\mathrm{Re}}$ is just a two-parameter regression, which is easier than Yuan or Jiang's three parameters regression processes.
TABLE 2: Parameters $A$ and $B$ regression based on Yuan and Jiang's experiment data.

\begin{tabular}{lcccc}
\hline Case & $\begin{array}{c}\varphi \\
\text { (shots/ft) }\end{array}$ & $\begin{array}{c}\theta \\
\left(^{\circ}\right)\end{array}$ & $A$ & $B$ \\
\hline 1 & 5 & 90 & 1.7872 & $0.873 N_{\mathrm{Re}}{ }^{-0.335}$ \\
2 & 5 & 180 & 1.8111 & $0.622 N_{\mathrm{Re}}{ }^{-0.295}$ \\
3 & 5 & 360 & 1.8460 & $0.651 N_{\mathrm{Re}}{ }^{-0.314}$ \\
4 & 10 & 90 & 3.0638 & $0.160 N_{\mathrm{Re}}{ }^{-0.170}$ \\
5 & 10 & 180 & 3.4043 & $0.360 N_{\mathrm{Re}}{ }^{-0.265}$ \\
6 & 10 & 360 & 4.9362 & $0.755 N_{\mathrm{Re}}{ }^{-0.290}$ \\
7 & 20 & 90 & 7.1489 & $1.471 N_{\mathrm{Re}}{ }^{-0.435}$ \\
8 & 20 & 180 & 8.3745 & $0.452 N_{\mathrm{Re}}{ }^{-0.270}$ \\
9 & 20 & 360 & 10.2128 & $1.078 N_{\mathrm{Re}}{ }^{-0.350}$ \\
\hline
\end{tabular}

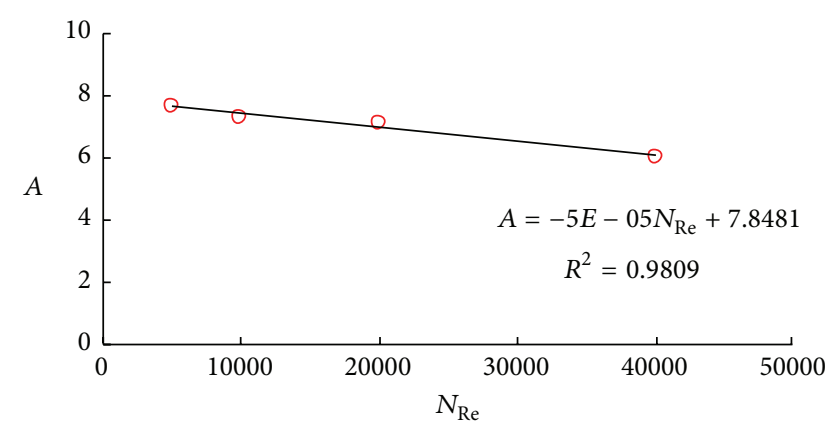

FIGURE 5: The relation between the line slope (parameter $A$ ) and the main flow rate (parameter $\left.N_{\mathrm{Re}}\right)$ in same case $\left(20\right.$ shots $\left./ \mathrm{ft}, 90^{\circ}\right)$.

For the case (20 shots $\left./ \mathrm{ft}, 90^{\circ}\right)$, the regression relation between $B$ and $N_{\operatorname{Re}}$ is given in (5) and Figure 6:

$$
B=1.4707 N_{\mathrm{Re}}^{-0.4345} \text {. }
$$

For $B=a N_{\mathrm{Re}}^{b}$, we can get the parameter $a=1.4707$ and $b=-0.4345$. The $B$ in different $N_{\mathrm{Re}}$ is listed in Table 1 .

Figure 6 shows the relation between $B$ and $N_{\mathrm{Re}}$ while 20 shots, $90^{\circ}$. It clearly presents the regression result and the well fit of the experiment data. Using the above method can obtain parameter $A$ and $B$ in (4) for other cases, which are listed in Table 2.

In Figure 7, for the same perforation shot phasing, parameter $A$ of apparent friction factor usually increases with the increase of completion shot density. The shot density and parameter $A$ have a good linear relation. It is evident that, 


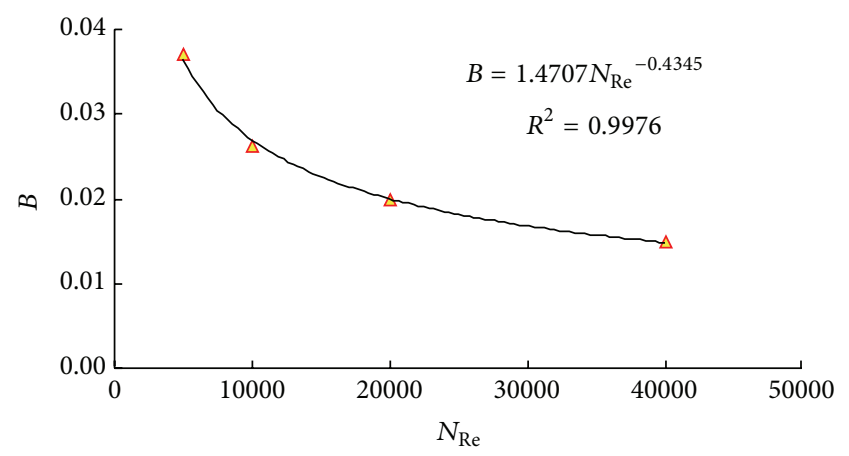

Figure 6: The relation between $B$ and $N_{\mathrm{Re}}$ for case $\left(20\right.$ shots/ $\left.\mathrm{ft}, 90^{\circ}\right)$.

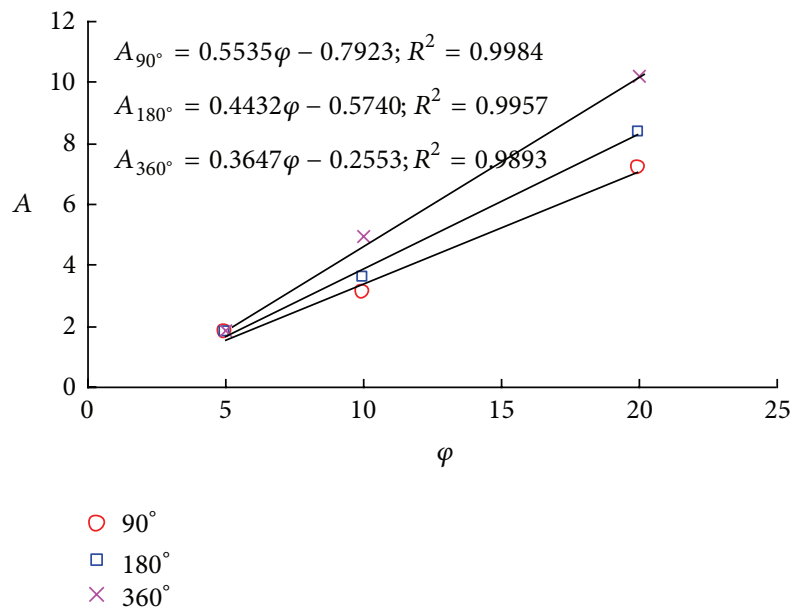

Figure 7: The relation between $A$ and $\varphi$ in different shot phasing $\theta$ $\left(90^{\circ}, 180^{\circ}\right.$, and $\left.360^{\circ}\right)$.

at high shot phasing, the shot density affects parameter $A$ obviously, while, at low shot phasing, the affection is only slightly.

Figure 8 shows that, for the same perforation shot density, parameter $A$ of the apparent friction factor is smaller when the phasing is lower. The shot phasing and parameter $A$ also has a good linear relation. Obviously, at high shot density, the shot phasing has more influence on parameter $A$ than it does at low shot density.

Based on the data shown in Figure 7, it is possible to work out the slopes and intercepts of the linear relation between $A$ and $\varphi$ at different shot phasing $\theta\left(90^{\circ}, 180^{\circ}\right.$, and $\left.360^{\circ}\right)$. Then the slopes and intercepts of parameter $A$ with different $\theta$ can be regressed. The regression results are presented in Figures 9 and 10.

Therefore, the parameter $A$ can be written as

$$
A=(0.0007 \theta+0.3110) \varphi+0.1833 \frac{360}{\theta}-1.0017 .
$$

Based on (3), $A$ can also be written in the flowing form:

$$
A=2 d \cdot f(\theta, \varphi) .
$$

As for all the above cases, the experimental facility used is a 4 $\mathrm{ft}$ long test section, which is a 1 in diameter horizontal pipe.

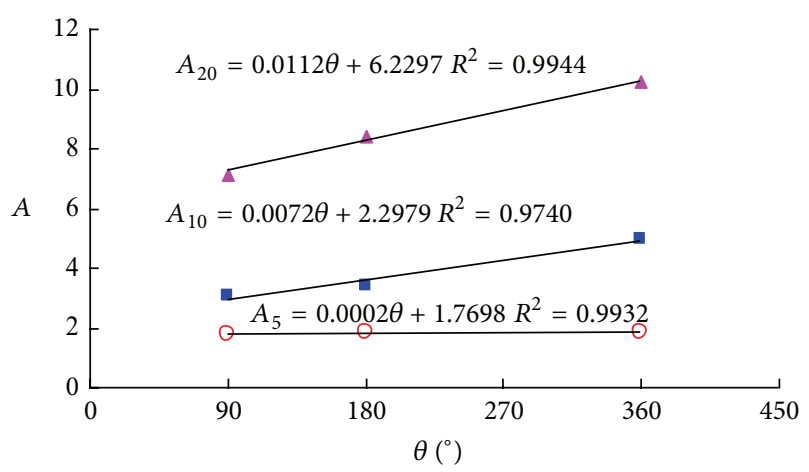

$$
\begin{aligned}
& \text { - } 5 \operatorname{shot} / f t \\
& \text { - } 10 \mathrm{shot} / \mathrm{ft} \\
& \text { - } 20 \mathrm{shot} / \mathrm{ft}
\end{aligned}
$$

Figure 8: The relation between $A$ and $\theta$ in different shot densities $\varphi$ $(5,10$, and 20 shots/ft).

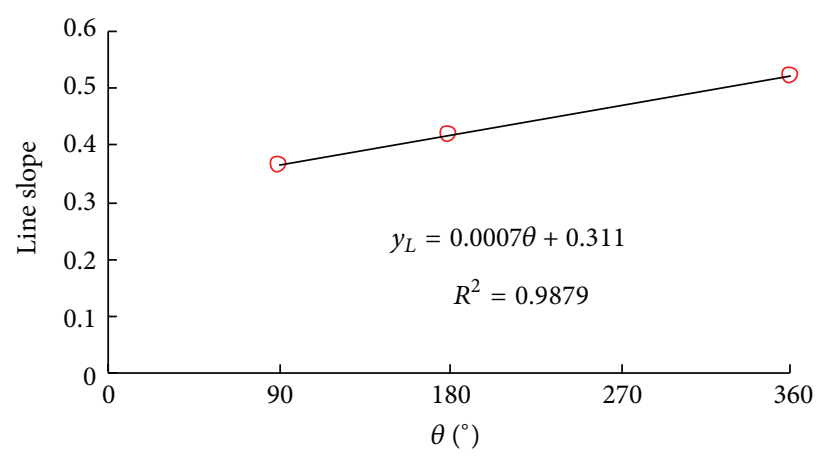

Figure 9: The slopes of the linear relation between $A$ and $\varphi$ at different shot phasing $\theta\left(90^{\circ}, 180^{\circ}\right.$, and $\left.360^{\circ}\right)$.

In SI unit system, $f(\theta, \varphi)$ can be written as

$$
f(a, \varphi)=(0.0035 \theta+1.866) \varphi+\frac{1300}{\theta}-20 .
$$

Therefore, the apparent friction factor can now be expressed as shown in (9) using the correlation factors such as shot density $\varphi$, shot phasing $\theta$, pipe diameter $d$, and main flow Reynolds number $N_{\mathrm{Re}}$ :

$$
\begin{aligned}
f_{t}\left(\theta, \varphi, d, N_{\mathrm{Re}}\right)= & {\left[(0.0035 \theta+1.866) \varphi+\frac{1300}{\theta}-20\right] \frac{2 d q}{\mathrm{Q}} } \\
& +f\left(N_{\mathrm{Re}}\right) .
\end{aligned}
$$

Examine the left side of (9), for different perforation shot densities and phasing, $f(\theta, \varphi)$ can be easily obtained, which is also the part that significantly affects $f_{t}$. For the contribution of $f\left(N_{\mathrm{Re}}\right)$, the regression results of the 9 cases are listed in Table 2. The values of parameter $B$ and $f\left(N_{\mathrm{Re}}\right)$ for other perforation shot densities and phasing that are not investigated in this research can be calculated through linear interpolation. 


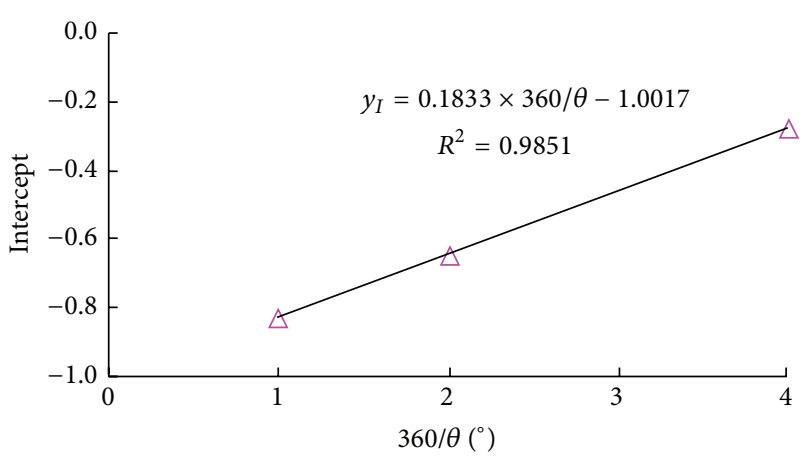

FIGURE 10: The intercepts of the linear relation between $A$ and $\varphi$ at different shot phasing $\theta\left(90^{\circ}, 180^{\circ}\right.$, and $\left.360^{\circ}\right)$.

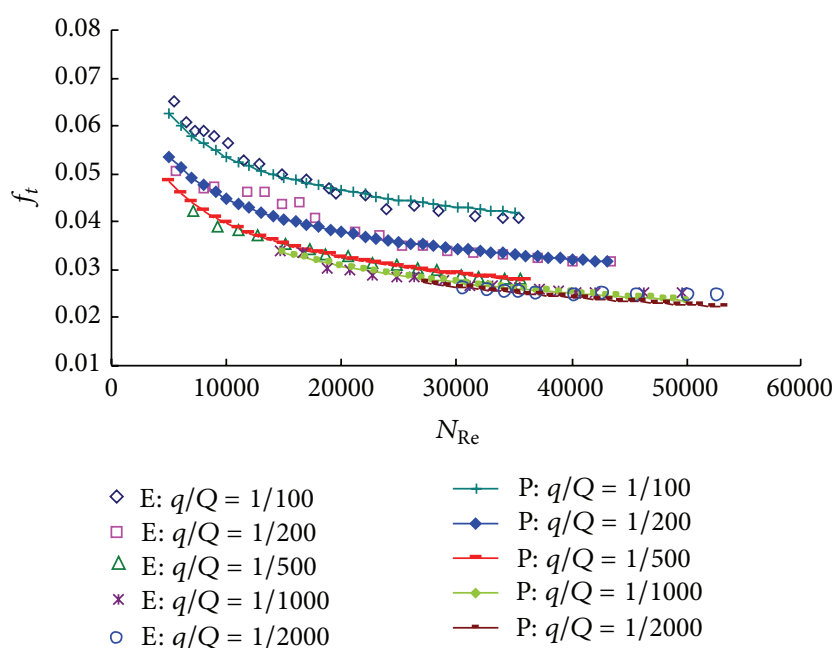

FIGURE 11: Comparison between new formula predictions and experiment data for the case of 5 shots $/ \mathrm{ft}$ and $360^{\circ}$ phasing.

\section{Data Comparison and Analysis}

In this section, the new apparent friction factor correlation formula (9) is further examined based on the study made by Yuan et al. [15] and Jiang et al. [17]. The focus was on data comparison between the prediction of (9) and the experiment data.

Figures 11, 12, and 13 show the variations in apparent friction factor with influx to main flow rate ratios and Reynolds numbers for the three test sections with shot pipe completions. Each figure is plotted as apparent friction factor $f_{t}$ versus Reynolds number $N_{\mathrm{Re}}$ and the data series represent experimental results at different influx to main flow rate ratios. "E" data series are the experiment data, while "P" series are the predicted data by (9). As it can be seen in Figures 1113 , there is a good match between the "E" and "P" data series, which in turn proves that (9) is a correct correlation for the theory of apparent wall friction factor and the experiment data.

Figure 14 illustrates the shot completion phasing affecting the apparent friction factor. The data are obtained under the condition that other parameters such as 20 shot/ft and $q / Q=$

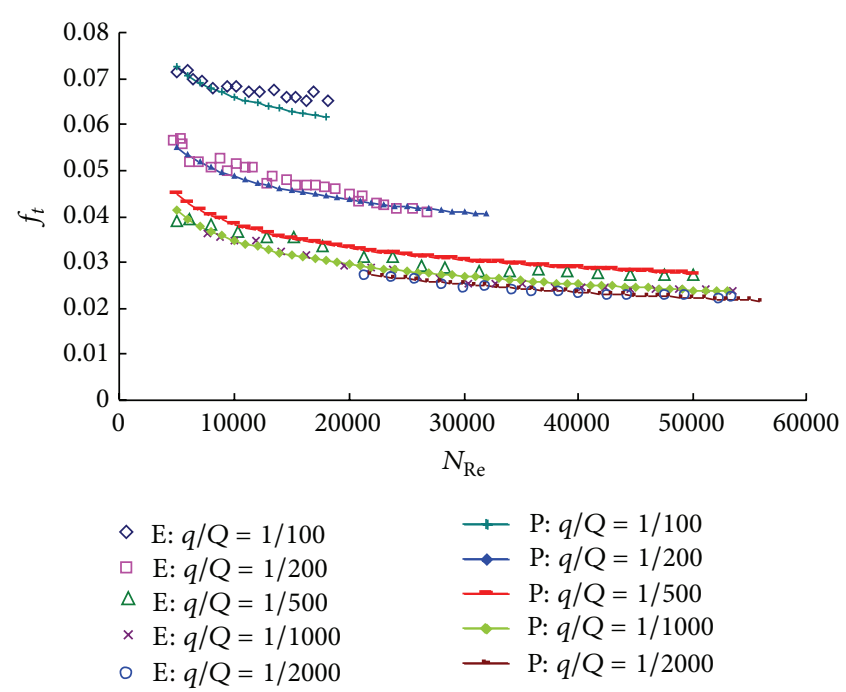

FIGURE 12: Comparison between new formula predictions and experiment data for the case of 10 shots/ft and $180^{\circ}$ phasing.

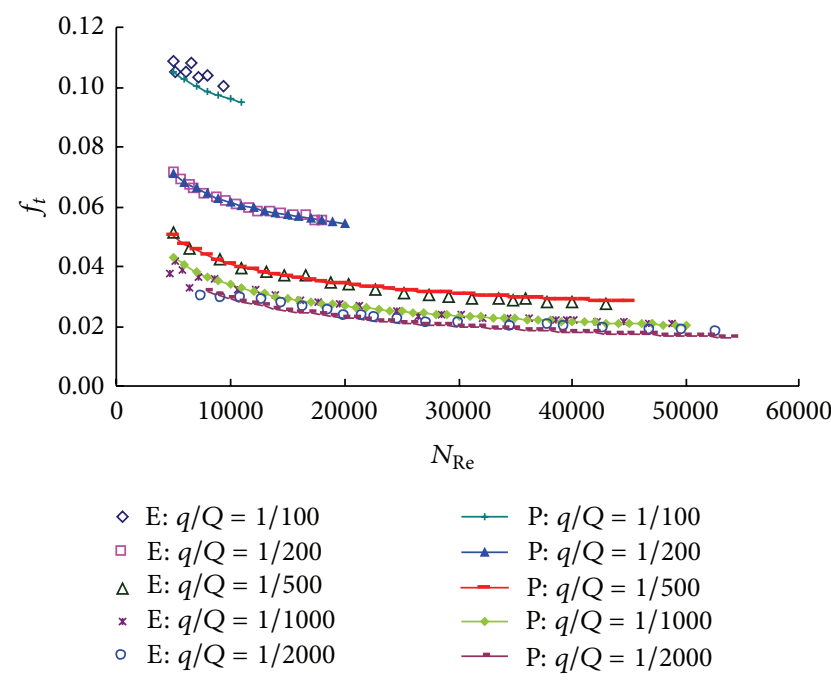

FIGURE 13: Comparison between new formula predictions and experiment data for the case of 20 shots $/ \mathrm{ft}$ and $90^{\circ}$ phasing.

$1 / 200$ are equal. As the completion phasing decreases from $360^{\circ}$ and $180^{\circ}$ to $90^{\circ}$, the apparent friction factor decreases. Again, the predictions made by the new formula, shown as "P" data series, match with the experiment data well.

Figure 15 presents the influence of the shot completion density on the apparent friction factor. The data were obtained under the condition that $180^{\circ}$ phasing and $q / Q=$ $1 / 200$ are used for each case. The increasing of the shot completion density from 5 to 10 and then to 20 shots/ft increases the apparent friction factor. The same result are obtained as in Figure 14; the predictions made by the new formula have good match with the experiment data. 


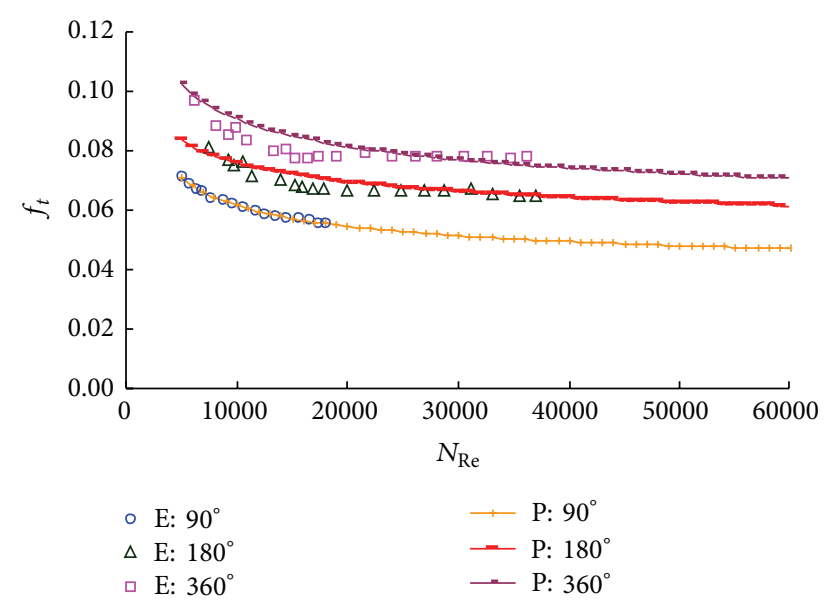

FIGURE 14: Comparison of experiment data and new prediction for three phasing for the case of 20 shot $/ \mathrm{ft}$ and $q / Q=1 / 200$.

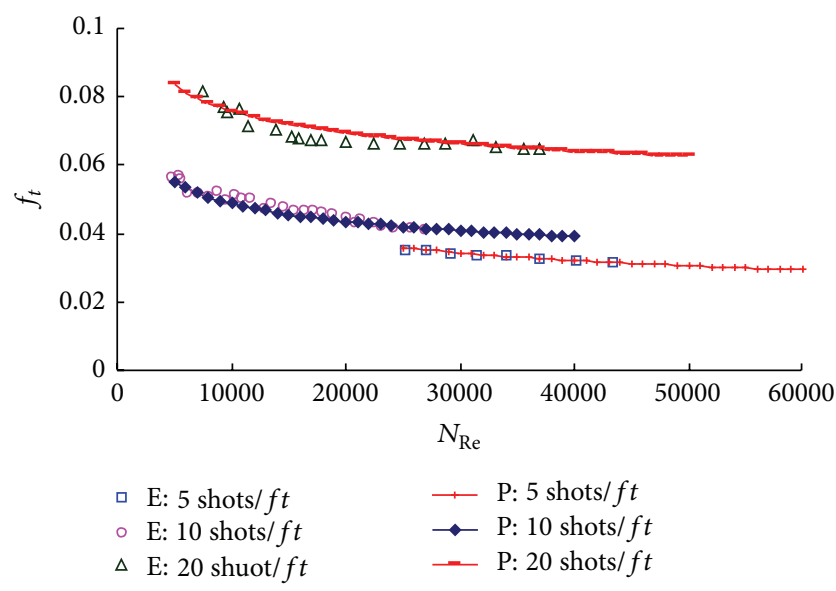

FIGURE 15: Comparison of experiment data and new prediction for different shot densities for the case of $180^{\circ}$ phasing and $q / Q=1 / 200$.

\section{Applications and Discussions}

The data by Yuan et al. [15, 16] and Jiang et al. [17] were obtained based on experiments in a small size pipe. Whether this result is applicable to practical field applications where the wellbore could be significantly larger than the pipe used in the experiments is uncertain. The new regression model presented in this paper has been applied to analyze Ouyang's example, a real horizontal well in an oil reservoir. The information of the case study is presented in Table 3, which is the same as used in Ouyang's [14] example except that SI system of units is used in this study rather than imperial system of units.

In Table 3, the partially perforated horizontal well means only a part of the wellbore is perforated for radial influx. Figures 16 and 17 are the graphics of pressure gradients and cumulative pressure drops from the start of the perforation in a partially perforated horizontal well obtained using different formulas.
TABLE 3: The information of horizontal wellbore, perforation, fluid, and flow rate.

\begin{tabular}{lc}
\hline Item & Value \\
\hline Horizontal wellbore length $(\mathrm{m})$ & 305 \\
Perforation length $(\mathrm{m})$ & 30.5 \\
Pipe ID $(\mathrm{m})$ & 0.157 \\
Relative pipe roughness & 0.0002 \\
Perforation ID $(\mathrm{m})$ & 0.0046 \\
Perforation density $($ shots $/ \mathrm{m})$ & 26 \\
Shots phasing $\left({ }^{\circ}\right)$ & 90 \\
Fluid density $\left(\mathrm{Kg} / \mathrm{m}^{3}\right)$ & 1000 \\
Fluid viscosity $(\mathrm{mPa} \cdot \mathrm{s})$ & 0.878 \\
Wellbore rate at perforation start point $\left(\mathrm{m}^{3} / \mathrm{s}\right)$ & 0.0129 \\
Radial influx rate $\left(\mathrm{m}^{3} / \mathrm{s}\right)$ & 0.0129 \\
\hline
\end{tabular}

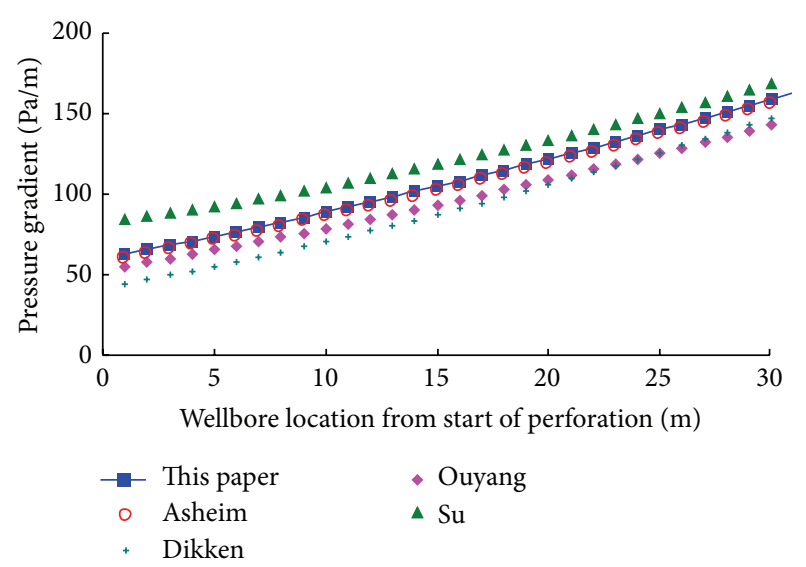

FIGURE 16: Comparison of pressure gradients using different formulas.

Dikken, Asheim, and Su's models did not consider the shot phasing impact on the pressure drop calculation. Also, it should be pointed out that no consideration was given to the perforation distribution in the wellbore flow modeling of the Dikken, Asheim et al. [7], and Su and Gudmundsson's [13] models. In Figures 16-17, Dikken's model gives the smallest values compared with other models. Dikken's model was established through constant resistance coefficient to calculate wellbore pressure drop; therefore, it can be understood that only the effect of wellbore friction pressure drop was considered. As a result, the outcome of Dikken's model is smaller than other models. Su's model pressure gradient predictive value is larger than other models. The main reason of this deviation is that the calculations of the perforation roughness pressure drop and the mixing pressure drop have partly superimposed. Based on Su's mixed pressure drop model, the pressure drop is too large. In Ouyang's study [14], it was found that the radial influx from the perforations reduces the wall friction for turbulent flow. The pressure drop only considered wall friction drop and acceleration drop and ignored the mixed pressure drop which is pressure gradient called inflow-directional-pressure gradient in Ouyang's paper caused by inflow direction. So, the result of Ouyang's model is 


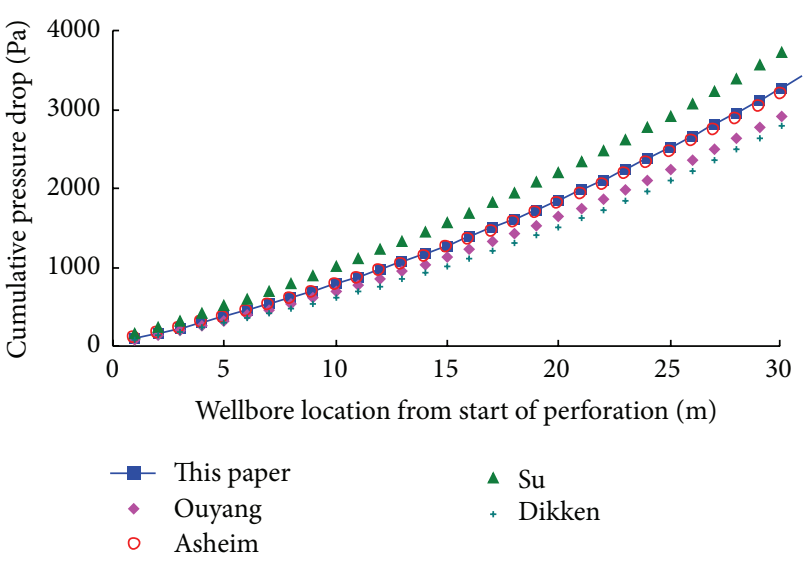

FIGURE 17: Comparison of cumulative pressure drops using different formulas.

smaller than other models except Dikken's. Ouyang's model gave the shot parameters (shot density and shot phasing) but did not have any calculations to show the impact of these parameters on the final result. The model presented by this paper is derived from the experimental data of Yuan's and part of Jiang et al.s [17]. The derivation process of this model is different from Asheim's process. But the calculated results are nearly the same when both models are applied to Ouyang's practical example. Compared with other models, the model produced by this research sits in the middle range as shown in Figures 16 and 17. The above analysis shows that Ouyang and Dikken models subpredict the pressure drop and Su's models overpredict the pressure drop. The model produced by this research and Asheim's models are within the lower and upper deviations. As the model presented in this paper regressed from Yuan and Jiang's experiment data, the analysis confirms that the outcome of the model is reasonable and has the potential to be applied for real cases.

\section{Conclusions}

A new regression model to calculate apparent friction factor for a single phase wellbore flow is established based on the experiment data from the literature. The apparent friction factor, which is incorporated not only with frictional and accelerational pressure drops but also with the pressure drop caused by radial influx, is readily applicable to different perforation parameters of shot such as shot phasing and density and also applicable to different fluid properties, pipe IDs, and main and radial influx rates.

For the same perforation shot phasing, apparent friction factor usually increases with the increasing of completion shot density. The shot density and apparent friction factor have a good linear relation. For the same perforation shot density, the apparent friction factor becomes smaller when the phasing gets lower. The shot phasing and apparent friction factor also have a good linear relation.

Compared with the models presented in literature, the outcome of the model established by this research is rational and realistic. Moreover, this new model makes it easy to calculate the pressure drop with different shot density and shot phasing with perforated completion. It is a model that can be easily implemented into reservoir simulators, analytical reservoir, and wellbore inflow coupling models.

\section{Nomenclatures}

$f_{t}:$ Apparent friction factor, Moody

$C_{n}:$ Coefficient in $f_{t}$

$d: \quad$ Pipe diameter, $\mathrm{m}$

A: $\quad$ Line slop of the relation between $f_{t}$ and $q / Q$

$B$ : Line intercept of the relation between $f_{t}$ and $q / Q$

a: The parameter of Blasius formula

$b$ : The parameter of Blasius formula

$\Delta x$ : Test pipe length, $\mathrm{m}$

$N_{\text {Re }}$ : Reynolds Number

$p_{1}$ : Upstream pressure, $\mathrm{Pa}$

$p_{2}$ : Downstream pressure, $\mathrm{Pa}$

Q: $\quad$ Main flow rate, $\mathrm{m}^{3} / \mathrm{s}$

$q$ : Volumetric influx flow rate from each injection opening, $\mathrm{m}^{3} / \mathrm{s}$

$\bar{U}: \quad$ Average main flow velocity, $\mathrm{m} / \mathrm{s}$

$\theta$ : Completion shot phasing,

$\varphi$ : $\quad$ Completion shot density, $1 / \mathrm{m}$

$\mu$ : $\quad$ Fluid viscosity, $\mathrm{mPa} \cdot \mathrm{s}$

$\rho: \quad$ Density, $\mathrm{kg} / \mathrm{m}^{3}$

$\beta_{2}$ : Momentum correction factor of upstream, $\mathrm{f}$

$\beta_{1}$ : Momentum correction factor of downstream, f.

\section{Conflict of Interests}

The authors declare that there is no conflict of interests regarding the publication of this paper.

\section{Acknowledgments}

This work was supported by the National Science and Technology Major Project of China (no. 2011ZX05010-002 and no. 2011ZX05024-003), the Innovation Foundation of Southwest Petroleum University (no. 2013XJZ004), and the Scientific Project of Sichuan Provincial Education Department (no. 14ZB0045).

\section{References}

[1] B. J. Dikken, "Pressure drop in horizontal wells and its effect on their production performance," in Proceedings of the 64th Annual Technical Conference and Exhibition, Paper SPE 19824, pp. 574-19824, San Antonio, Tex, USA, October 1989.

[2] G. T. Jackson, M. T. Balhoff, C. Huh, and M. Delshad, "CFDbased representation of non-Newtonian polymer injectivity for a horizontal well with coupled formation-wellbore hydraulics," Journal of Petroleum Science and Engineering, vol. 78, no. 1, pp. 86-95, 2011.

[3] D. Y. Ding, "Coupled simulation of near-wellbore and reservoir models," Journal of Petroleum Science and Engineering, vol. 76, no. 1-2, pp. 21-36, 2011. 
[4] R. M. S. M. Schulkes and O. H. Utvik, "Pressure drop in a perforated pipe with radial inflow: single-phase flow," SPE Journal, vol. 3, no. 1, pp. 77-85, 1998.

[5] H. Yuan, C. Sarica, and J. P. Brill, "Effect of perforation density on single phase liquid flow behavior in horizontal wells," in Proceedings of the 2nd SPE International Conference on Horizontrd Well Technology, SPE 37109, pp. 603-612, Alberta, Canada, November 1996.

[6] Z.-M. Wang, J.-N. Xiao, X.-Q. Wang, and J.-G. Wei, "Experimental study for pressure drop of variable mass flow in horizontal well," Journal of Experiments in Fluid Mechanics, vol. 25, no. 5, pp. 26-29, 2011 (Chinese).

[7] H. Asheim, J. Kolnes, and P. A. Oudeman, "A flow resistance correlation for completed wellbore," Journal of Petroleum Science and Engineering, vol. 8, no. 2, pp. 97-104, 1992.

[8] R. A. Novy, "Pressure drops in horizontal wells: when can they be ignored?" SPE Reservoir Engineering, vol. 10, no. 1, pp. 29-35, 1995.

[9] E. Ozkan, C. Sarica, M. Haciislamoglu, and R. Raghavan, "The influence of pressure drop along the wellbore on horizontal well productivity," in Proceedings of the SPE Production Operations Symposium, Paper SPE 25502, Stillwater, Okla, USA, March 1993.

[10] E. Ozkan, C. Sarica, M. Haciislamoglu, and R. Raghavan, "Effect of conductivity on horizontal well pressure behavior," Paper SPE 30230 as supplement to paper 24683, 1995.

[11] B. Guo, "Corrections to horizontal drain hole productivity equations for wellbore friction effect," Journal of Petroleum Science and Engineering, vol. 70, no. 3-4, pp. 344-349, 2010.

[12] J.-H. Zhang, C.-Q. Yan, and P.-Z. Gao, "Characteristics of pressure drop and correlation of friction factors for single-phase flow in rolling horizontal pipe," Journal of Hydrodynamics B, vol. 21, no. 5, pp. 614-621, 2009.

[13] Z. Su and J. S. Gudmundsson, "Pressure drop in perforated pipes: experiments and analysis," in Proceedings of the Asia Pacific Oil \& Gas Conference, Paper SPE 28800, pp. 563-574, Melbourne, Australia, November 1994.

[14] L.-B. Ouyang, S. Arbabi, and K. Aziz, "General wellbore flow model for horizontal, vertical and slanted well completions," SPE Journal, vol. 3, no. 2, pp. 124-132, 1998.

[15] H. Yuan, C. Sarica, S. Miska, and J. P. Brill, "An experimental and analytical study of single-phase liquid flow in a horizontal well," Journal of Energy Resources Technology, vol. 119, no. 20, pp. 20-25, 1997.

[16] H. Yuan, C. Sanica, and J. P. Brill, "Effect of completion geometry and phasing on single-phase liquid flow behavior in horizontal wells," in Proceedings of the SPE Technical Conference and Exhibition, Paper SPE 48937, pp. 93-104, New Orleans, Louisiana, September 1998.

[17] W. Jiang, C. Sarica, E. Ozkan, and M. Kelkar, "Investigation of the effects of completion geometry on single-phase liquid flow behavior in horizontal wells," Journal of Energy Resources Technology, vol. 123, no. 2, pp. 119-126, 2001.

[18] W. Jiang, Investigation of the effects of completion geometries upon single phase liquid flow behavior in horizontal wells [M.S. thesis], The University of Tulsa, Tulsa, Okla, USA, 1999. 


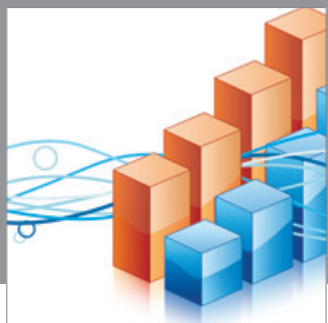

Advances in

Operations Research

mansans

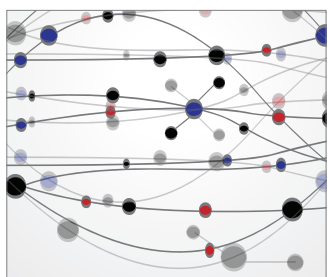

The Scientific World Journal
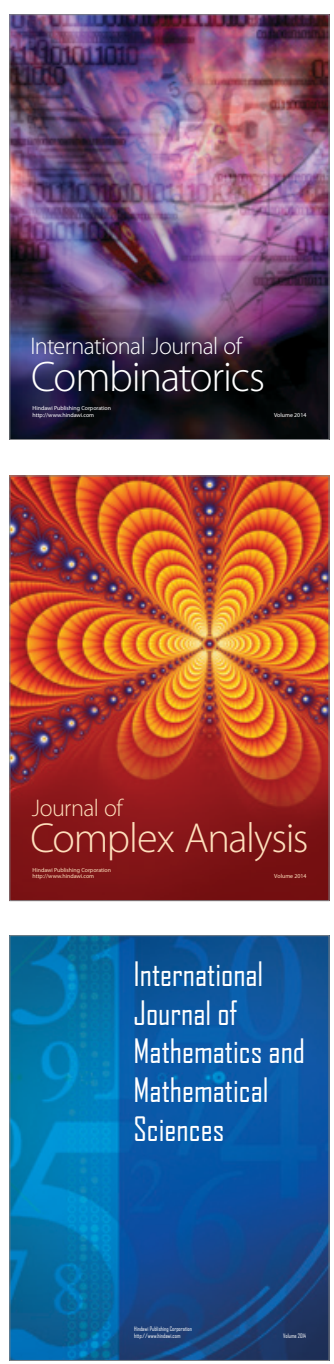
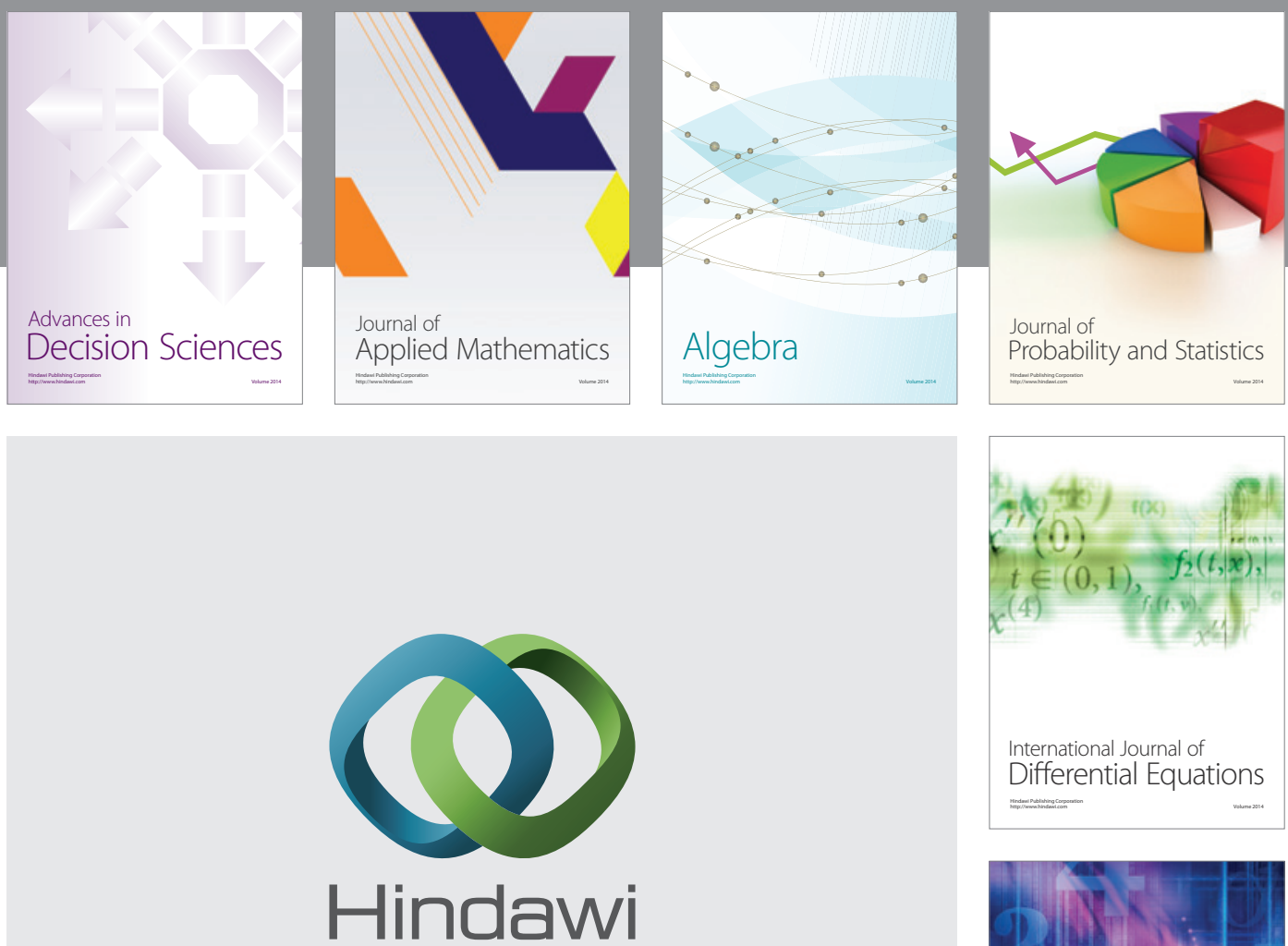

Submit your manuscripts at http://www.hindawi.com
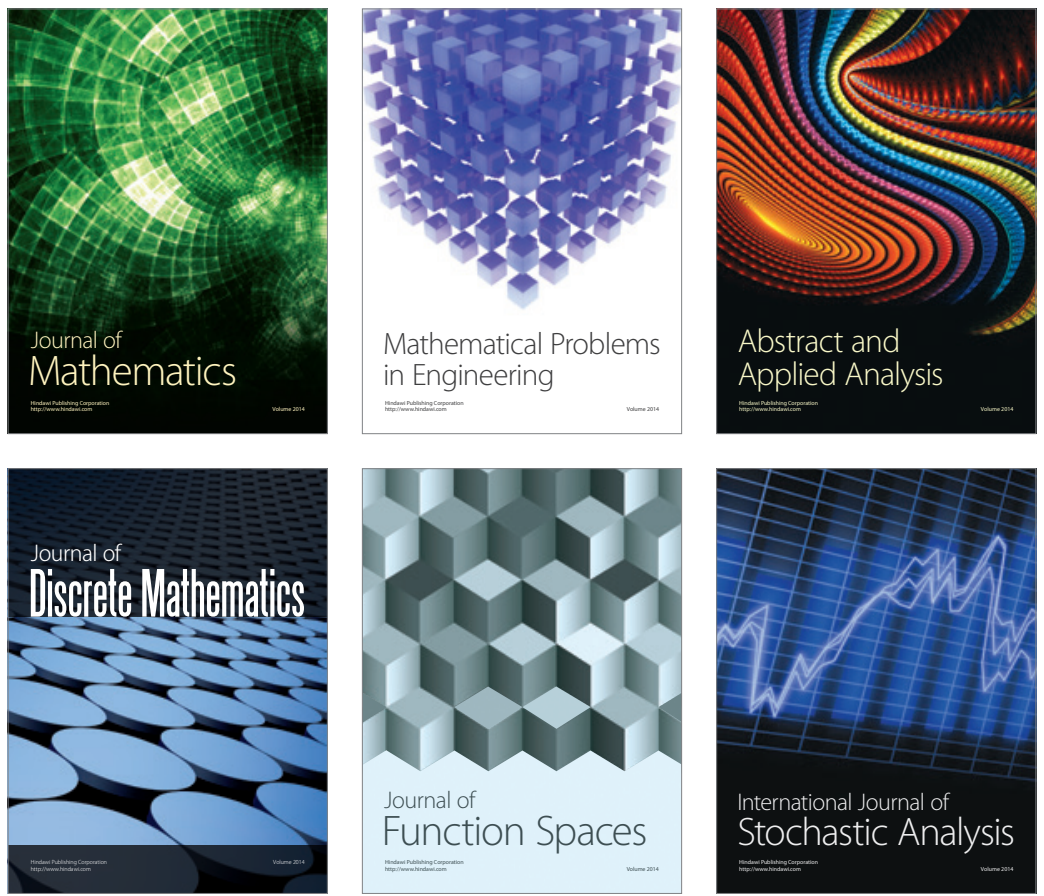

Journal of

Function Spaces

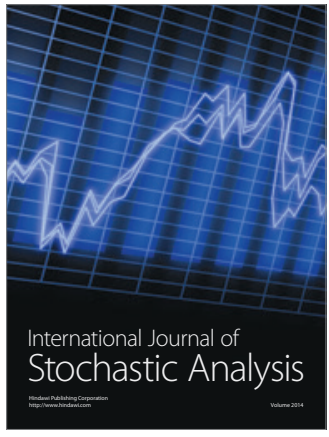

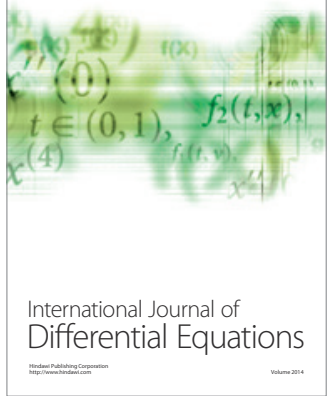
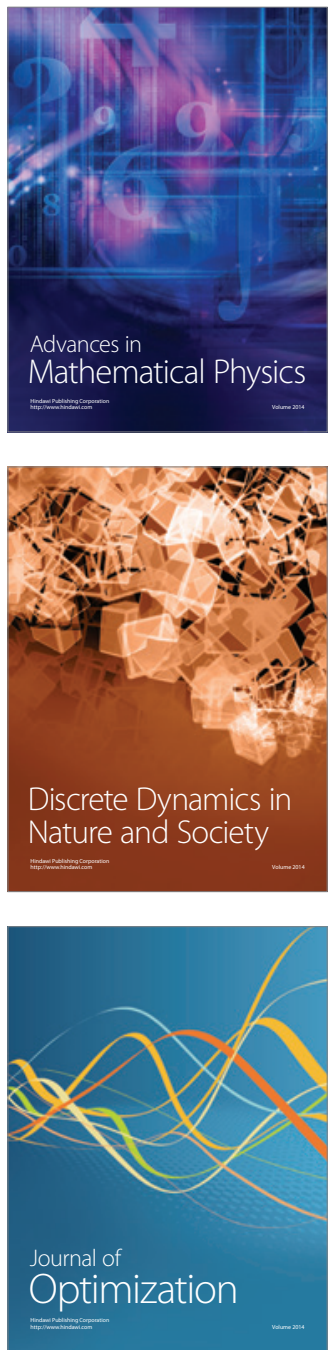